\title{
Label-Free Quantitation: A New Glycoproteomics Approach
}

\author{
Kathryn R. Rebecchi, Jamie L. Wenke, Eden P. Go, and Heather Desaire \\ Department of Chemistry, University of Kansas, Lawrence, Kansas, USA
}

We demonstrate herein a method for quantifying glycosylation changes on glycoproteins. This novel method uses MS data of characterized glycopeptides to analyze glycosylation profiles, and several quality control tests were done to demonstrate that the method is reproducible, robust, applicable to different types of glycoproteins, and tolerant of instrumental variability during ionization of the analytes. This method is unique in that it is the first label-free quantitative method specifically designed for glycopeptide analysis. It can be used to monitor changes in glycosylation in a glycosylation site-specific manner on a single glycoprotein, or it can be used to quantify glycosylation in a glycoprotein mixture. During mixture analysis, the method can discriminate between changes in glycosylation of a given protein, and changes in the glycoprotein's concentration in the mixture. This method is useful for quantitative analyses in biochemical studies of glycoproteins, where changes in glycosylation composition can be linked to functional differences; it could also be implemented in the pharmaceutical industry, where glycosylation profiles of glycoprotein-based therapeutics must be quantified. Finally, quantification of glycopeptides is an important aspect of glycopeptide-based biomarker discovery, and our quantitative approach could be a valuable asset to this field as well, provided the compositions of the glycopeptides to be quantified are identifiable using other methods. (J Am Soc Mass Spectrom 2009, 20, 1048-1059) (c) 2009 Published by Elsevier Inc. on behalf of American Society for Mass Spectrometry

G lycoproteomics, the study of the glycome attached to proteins, is a vital research field because as many as $50 \%$ of proteins in the human body are glycosylated [1]. Glycoproteins are not only common, but the glycans on the proteins are significant because they are known to play important biological roles in the body, including cell-cell interaction, cell recognition, and protein regulation [2]. While proteins are genetically encoded, the glycosylation on proteins depends on the glycosylating enzymes that are present and the local cell environment. Therefore, the amount of enzymes and cofactors involved in glycosylation affect the extent of glycosylation on the glycoprotein [3]. Changes in glycosylation are known to occur with the onset of certain diseases such as cancer [4-6]. Thus, detection methods to monitor changes in glycosylation of glycoproteins are essential to determine possible biomarkers for cancer and other diseases.

Methods to monitor changes in glycosylation of proteins are not just important for biomarker studies. These methods are also important for pharmaceutical development, since glycoproteins have become increasingly desirable targets as therapeutic agents. Some example glycoprotein-based pharmaceuticals include erythropoietin [7], follicle stimulating hormone [8], thy-

Address reprint requests to Dr. H. Desaire, Department of Chemistry, University of Kansas, 1251 Wescoe Hall Drive, Lawrence, KS 66045. E-mail: hdesaire@ku.edu roid stimulating hormone [9], and vaccine candidates, such as the heavily glycosylated envelope glycoprotein on the surface of the HIV virus [10-12]. Profiling and quantifying the glycosylation on these products is important because studies indicate that glycosylation in cell expression systems can differ from the glycosylation that occurs in the human body [10], and researchers are currently striving to overcome this problem by modifying the glycosylation, or humanizing it, during protein production [13, 14]. Therefore, as methods are developed for humanizing glycosylation, a quantitative method that distinguishes between glycosylation profiles on native and modified glycoproteins is imperative.

There are two options for quantifying glycosylation on proteins: quantifying the glycans after enzymatic or chemical cleavage from the protein or quantifying glycopeptides. While glycan analysis is clearly a more established technique [15-18], this approach restricts the amount of information one can obtain about the glycosylation profile. For example, in purified, multiply glycosylated proteins, the study of released glycans would only provide aggregate information about the glycosylation on a protein, and it would not provide information about the glycosylation profile at a specific glycosylation site. Yet it is well established that monitoring glycosylation profiles at individual glycosylation sites is important because the glycosylation at particular sites in a protein can modulate the protein's structure, function, or metabolic clearance [19]. Since many ther- 
apeutic proteins are multiply glycosylated, including all the examples mentioned above, the analysis of released glycans is problematic in that it does not provide glycosylation site-specific information about the glycosylation profiles of the protein.

In biomarker analysis, quantifying the released glycans has an additional disadvantage in that all the information about the proteins from which the glycans originated is lost. This introduces many problems in biomarker discovery, such as not being able to identify whether or not the glycan's concentration increased because a protein containing that glycan was overexpressed, or if one or several proteins' glycosylation profile changed, causing the glycan to be more abundant, even though the protein level(s) are not altered [4]. In contrast to glycan analysis, glycopeptide analysis provides glycosylation site-specific information for purified proteins $[17,20,21]$, and it could potentially be useful in distinguishing between glycosylation changes and protein expression changes, since the protein information is encoded in the glycopeptide. For the reasons described herein, we are pursuing quantitative methods for glycopeptides.

There are two strategies to quantify changes in the glycosylation of proteins, either differentially labeling sets of samples or using label-free approaches. Several labeling techniques exist, including those with detection by optical methods [22, 23] and mass spectrometry [24-26]. One common quantitation strategy using labeling and optical detection involves the use of lectins to bind glycoproteins in complex samples, then detecting different types of glycosylation due to differential binding of the lectins. Because different lectins have different specificities for classes of N-linked glycans, it is possible to use lectins to distinguish between high mannose and complex type glycosylation, for example. Detecting the binding of lectins to glycans, glycopeptides, or glycoproteins is done by either tagging the lectins [22, 27] or tagging the analyte [23] with a fluorophore, followed by the monitoring of a change in fluorescence upon binding. If the ultimate quantitative goal is to detect changes in classes of N-linked glycans, optical methods that detect differences in lectin binding are ideal and have very low detection limits. However, the use of lectin microarrays is incapable of observing subtle glycosylation changes, such as a change in the number of mannoses present on a high mannose glycan [23].

If detecting subtle changes in glycosylation is required, such as distinguishing between the addition or subtraction of one monosaccharide unit between glycoprotein samples, other detection strategies, for example mass spectrometry, must be employed. Quantitative MS analysis of glycosylated species using isotopic labels is a growing field. Currently, methods are available to analyze glycans directly [25, 26, 28-31], and the strategy used in some of these approaches could potentially be applied to glycopeptide analysis. The biggest draw-back is that many of these labeling methods are also limited to two sample sets, a control group and a test group; therefore, if more samples need to be compared to one another, such as analyzing the glycosylation differences between five different vaccine candidates, binary labeling approaches become difficult to implement.

An alternative strategy for accomplishing a quantitative analysis is to use label-free approaches. These methods have the potential to compare multiple samples with ease. Changes in intensities of mass spectral peaks have been assessed by comparing different sample sets through either glycan analysis [4] or glycopeptide analysis [32]. Because signal intensities can vary between mass spectrometric samples, label-free approaches are not as commonly used for quantitative analysis [26, 33].

To alleviate much of the variation due to changes in MS response among samples, normalization of data has been applied in proteomics studies [33-36]. Many proteomics researchers employ a normalization technique that involves dividing intensities of individual peaks by the total intensity from all peaks in the spectrum [33-35]; this approach is fundamentally similar to the approach by Wang et al., where spectra are normalized by calculating an intensity ratio for one spectrum versus another by doing pair-wise comparisons of peak intensities, for all the peaks in the spectra [36]. In the work presented here, this concept of normalization is builtupon to produce a label-free quantitative method for glycopeptide analysis. While in the proteomic field one can obtain reliably quantitative data by normalizing the data to the total ion abundance [33-35], this method is potentially problematic for glycopeptide analysis because the glycopeptides ionize weakly, compared with the nonglycosylated peptides that may also be present. Therefore, small changes in the presence of nonglycosylated interferents could impart large variability in a quantitative assay when the total ion current (or pairwise comparison of all the peaks) is used to normalize the ion abundances of the analytes. To remedy this problem, a new normalization method is described herein, where the ion abundance from each glycopeptide is divided by the total intensity of all glycopeptide peaks present in a given spectrum (excluding all ions that are not assigned as glycopeptides). As demonstrated herein, this normalization produces reproducibly quantitative, label-free data.

The second major innovation of this work is using a two-tiered quantitative analysis. In the first tier of the analysis, the abundances of glycopeptide ions within a given sample are compared to each other. This internal analysis is used to generate a glycosylation profile for the sample, where the abundance of each glycoform is rank-ordered (from smallest to largest) within the sample. The second tier of the analysis involves comparing this generated glycopeptide profile from one sample to the profile of another sample. By comparing whole profiles, and not just the abundance of a given glycoform, one can readily discriminate between changes in the overall glycosylation profile of a protein and changes 
in the abundance of a given glycoprotein present in a mixture of other species. The described method, which presents a new normalization method custom-designed for the challenges of glycopeptide analysis, and a new approach to glycosylation profiling, where internal and external analyses are completed in parallel, is useful for those interested in glycosylation profiling of biopharmaceuticals as well as those quantifying mixtures of glycoproteins for various applications, including biomarker analysis.

\section{Experimental}

\section{Materials and Reagents}

All reagents were obtained in high purity from Sigma Aldrich except when noted otherwise. Ribonuclease B (RNase B) $>80 \%$ pure, asialofetuin, urea, $\alpha$-mannosidase from Canavalia ensiformis, dithiothreitol (DTT), iodoacetamide (IAA), acetic acid, Sepharose CL-4B, HPLC grade 1-butanol, and HPLC grade ethanol were all purchased from Sigma Aldrich (St. Louis. MO). Sequencing grade modified trypsin was purchased from Promega (Madison, WI). Ammonium bicarbonate $\left(\mathrm{NH}_{4} \mathrm{HCO}_{3}\right)$ was purchased from Fluka (Milwaukee, WI). HPLC grade methanol was purchased from ThermoFisher Scientific (Fairlawn, NJ). Water was purified by a Millipore Direct Q-3 water purification system (Billerica, MA).

\section{Enzymatic Glycan Trimming with $\alpha$-Mannosidase}

To analyze glycosylation change, one RNase B sample was subjected to cleavage by the enzyme $\alpha$-mannosidase as described by Toumi and Go [37]. Briefly, $\sim 300 \mu \mathrm{g}$ of RNase $B$ was dissolved in enough $10 \mathrm{mM} \mathrm{NH}_{4} \mathrm{HCO}_{3}$ (pH 5.0) to make a $2 \mathrm{mg} / \mathrm{mL}$ solution. The enzyme, $\alpha$-mannosidase, was added in an enzyme:protein ratio of 1:1000 (mol/ $\mathrm{mol})$. The sample was allowed to incubate for $24 \mathrm{~h}$ at $37^{\circ} \mathrm{C}$. Enough $\mathrm{NaOH}$ was added to raise the $\mathrm{pH}$ of the sample to approximately $\mathrm{pH}$ 8.0. The sample was then digested with trypsin, and subjected to glycopeptide enrichment, mass spectrometry, and data analysis, as described below.

\section{Glycoprotein Protease Digestion}

Approximately $300 \mu \mathrm{g}$ of glycoprotein was dissolved in $25 \mathrm{mM} \mathrm{NH}_{4} \mathrm{HCO}_{3}$ (pH 7.5-8.0) containing $4 \mathrm{M}$ urea, to a glycoprotein concentration of $1 \mathrm{mg} / \mathrm{mL}$ (asialofetuin), or $2 \mathrm{mg} / \mathrm{mL}$ (RNase B). To this solution, dithiothreitol (DTT) was added to a final concentration of $15 \mathrm{mM}$, and it was incubated at room temperature for $1 \mathrm{~h}$. Following the incubation, iodoacetamide (IAA) was added to a final concentration of $25 \mathrm{mM}$, and the reaction was stored at room temperature, in the dark, for $1 \mathrm{~h}$. Additional DTT was added to a final concentration of $40 \mathrm{mM}$ to neutralize excess IAA. The solution was diluted with $25 \mathrm{mM} \mathrm{NH}_{4} \mathrm{HCO}_{3}$ until the urea concentration was less than $1 \mathrm{M}$. Trypsin was added at a 1:50 (wt/wt) protease/glycoprotein ratio. The solution was allowed to incubate at $37^{\circ} \mathrm{C}$ for $18 \mathrm{~h}$ and stopped by the addition of $1 \mu \mathrm{L}$ acetic acid per $100 \mu \mathrm{L}$ solution.

\section{Glycopeptide Enrichment}

To remove the nonglycosylated peptides from samples, a method adapted from Wada et al. [38, 39] was used as also described by Zhang et al. [40]. Briefly, the digest solution was added to $800 \mu \mathrm{L}$ of $5: 1: 1$ (vol/vol) 1-butanol/ethanol/water and $25 \mu \mathrm{L}$ SepharoseCL-4B, and shaken gently for 45 min before centrifugation and extraction of the solution layer. Samples were washed twice with the addition of $1 \mathrm{~mL}$ 5:1:1 (vol/vol) 1-butanol/ethanol/water with gentle shaking for $5 \mathrm{~min}$ followed by the same centrifugation and extraction. After washing, $1 \mathrm{~mL}$ of 1:1 (vol/vol) ethanol/water was added, and samples were allowed to stand for $30 \mathrm{~min}$, followed by gentle shaking for $30 \mathrm{~min}$. The samples were centrifuged, and the solution layer was extracted and collected. The ethanol/water extraction step was repeated a second time. The combined samples were dried using a Labconco centrivap cold trap (Kansas City, $\mathrm{MO}$ ) and stored at $-20{ }^{\circ} \mathrm{C}$ until use.

For the quality control studies, the samples were reconstituted before MS analysis in 1:1 (vol/vol) water/ methanol containing $0.5 \%$ acetic acid, to a final glycopeptide concentration of $10 \mu \mathrm{M}$. After the initial MS analysis, the remaining RNase $\mathrm{B}$ was stored in the reconstituted solvent at $-20^{\circ} \mathrm{C}$ for $8 \mathrm{wk}$ for a second MS analysis testing the method's robustness.

For the mixture analysis experiments, RNase B and asialofetuin glycopeptide digest samples were each

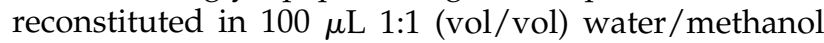
containing $0.5 \%$ acetic acid. The reconstituted samples of asialofetuin and RNase B glycopeptides were combined into four separate vials in varying concentrations. A total of four samples were prepared where the asialofetuin glycopeptides retained a fixed concentration of $1 \mu \mathrm{M}$, and the RNase B glycopeptide concentration prepared at $1 \mu \mathrm{M}, 3 \mu \mathrm{M}, 5 \mu \mathrm{M}$, and $10 \mu \mathrm{M}$, respectively, in each mixture vial (see Table 1).

\section{Mass Spectrometry}

MS and MS/MS data were acquired on a Thermo electrospray ionization-linear ion trap-Fourier transform ion cyclotron resonance (ESI-LIT-FTICR) mass spectrometer, (San Jose, CA), containing a 7 Tesla actively shielded magnet. The samples were injected by direct infusion at a flow rate of $1 \mu \mathrm{L} / \mathrm{min}$ in positive ion mode. The spray voltage was optimized to maximize

Table 1. Glycoprotein mixture concentrations

\begin{tabular}{lcccr}
\hline Sample no. & 1 & 2 & 3 & 4 \\
\hline \hline RNase B & $1 \mu \mathrm{M}$ & $3 \mu \mathrm{M}$ & $5 \mu \mathrm{M}$ & $10 \mu \mathrm{M}$ \\
Asialofetuin & $1 \mu \mathrm{M}$ & $1 \mu \mathrm{M}$ & $1 \mu \mathrm{M}$ & $1 \mu \mathrm{M}$ \\
\hline
\end{tabular}


ion signal, and the value ranged between 2.8 and $4.0 \mathrm{kV}$. The nebulizing gas, $\mathrm{N}_{2}$, was set to $10 \mathrm{psi}$, and the capillary temperature was $200{ }^{\circ} \mathrm{C}$. MS data were acquired with 100,000 resolutions for $\mathrm{m} / \mathrm{z} 400$, over a mass range of $m / z 500-2000$ for RNase $B$ and a mass range of $m / z$ 800-2000 for asialofetuin. For all MS ${ }^{1}$ data, 50 scans (with each containing 10 microscans) were averaged. For MS/MS data, the precursor ion was isolated with a $2 \mathrm{Da}$ isolation range; the activation time was set to 30 ms, the activation $q_{z}$ was 0.250 , and the activation energy was $30 \%$, as defined by the instrument software. There were 20 to 30 scans (each containing $10 \mathrm{mi}-$ croscans) averaged during acquisition of MS/MS data. The instrument software used was Xcalibur version 1.4 SR1 (ThermoFisher Scientific, San Jose, CA).

\section{Data Analysis}

The glycopeptide ions were assigned by matching theoretical masses to the actual masses acquired in the MS data. Prediction tables of possible theoretical glycopeptide masses were constructed for each glycoprotein studied. The prediction table was generated by the following steps: the amino acid sequence of each protein was obtained from Uniprot (http://beta.uniprot. org), and a theoretical tryptic digest of the given glycoprotein was completed by importing that sequence into Protein Prospector (http:/ / prospector.ucsf. edu/), which calculates the possible tryptic fragments. The mass of the resulting tryptic fragments were adjusted to account for the alkylation of cysteine residues by iodoacetamide. The peptide masses that contained glycosylation sites were added to possible N-linked glycan masses to give predicted glycopeptide masses. The glycan masses used in this case were the known glycans that are appended to these proteins, as described in references [41, 42], for asialofetuin and RNase $\mathrm{B}$, respectively. After combining the peptide masses with the known glycan masses, the calculated glycopeptide masses were converted to $m / z$ (for the $+1,+2$, $+3,+4$, and +5 charge states) for comparison with the $\mathrm{MS}^{1}$ data. A maximum of two missed tryptic cleavages was considered, as well as the presence of protonated and sodiated glycopeptide peaks. Possible peak identities from the MS data were confirmed through analysis of MS/MS data taken on each peak in the spectrum, as described previously [43].

\section{Data Treatment for Quantitative Method}

After identifying all the glycopeptide peaks in the spectrum, six steps were taken to process the data for quantitative analysis. The steps were (1) peak lists of $\mathrm{m} / \mathrm{z}$ and intensity were generated in Xcalibur, and transferred to Microsoft Excel; (2) the first four isotopic peaks of each glycopeptide ion were summed to obtain each glycopeptide's peak intensity; (3) glycopeptide peak intensities from all glycopeptide peaks in a spectrum were summed to calculate the total glycopeptide intensity. (4) The glycopeptide peak percentage was computed by eq 1 , below. This percentage reports how large each glycopeptide peak is, relative to the total glycopeptide intensity.

$$
\begin{aligned}
& \text { Glycopeptide Peak \% } \\
& =\frac{\text { Glycopeptide Peak Intensity }}{\text { Total Glycopeptide Intensity }} \times 100
\end{aligned}
$$

(5) Glycopeptide peak percentages corresponding to the same glycopeptide composition, but containing different charge states and/or charge carriers, were summed to give the glycopeptide percentage. If glycopeptides from a given glycosylation site also were generated with differing levels of missed tryptic cleavages, these species were also combined into one glycopeptide percentage. This percentage is a measure of how abundant a given glycopeptide composition is in the sample, regardless of whether or not it ionizes as a single peak, or as several peaks corresponding to different charge states, different numbers of sodiated adducts, or different lengths of peptide, due to missed tryptic cleavage; (6) glycopeptide percentages were rank ordered to determine which glycopeptides were most abundant in the spectrum, and the rank order among different samples was compared.

\section{Results and Discussion}

\section{Developing a Quantitative Method for Glycopeptides}

Since the goal is to develop a label-free quantitative approach for glycopeptides, the first problem that must be overcome is the fact that ion abundances in mass spectra are not very reproducible in run-to-run analyses. Figure 1 demonstrates this by showing MS data of glycopeptides generated from two replicate samples of asialofetuin digested with trypsin. The brown and red stars in Figure 1 label peaks that correspond to two different charge states of a single glycopeptide composition, the $4^{+}$and $5^{+}$charge states, respectively. The intensity of the peaks labeled with the brown stars does not change between Figure $1 \mathrm{a}$ and $\mathrm{b}$ because this is the base peak in both spectra. The peaks labeled with red stars, on the other hand, have a much higher intensity in Figure 1a compared with Figure 1b, demonstrating that the ions partitioned differently into different charge states in the two analyses. The insets in Figure $1 \mathrm{a}$ and $\mathrm{b}$ show the intensities of sodiated adducts are also different when the two spectra are compared. The peaks labeled with green stars, which correspond to sodiated adducts of some of the glycopeptides, have much lower intensities in Figure $1 \mathrm{~b}$ compared with Figure 1a. Consequently, the distribution between charge states and sodiated adducts of the glycopeptide peaks in Figure 1a is different than the distribution of the glycopeptide peaks in Figure $1 \mathrm{~b}$. Therefore, it makes sense that the peaks from Figure $1 a$ and $b$ 


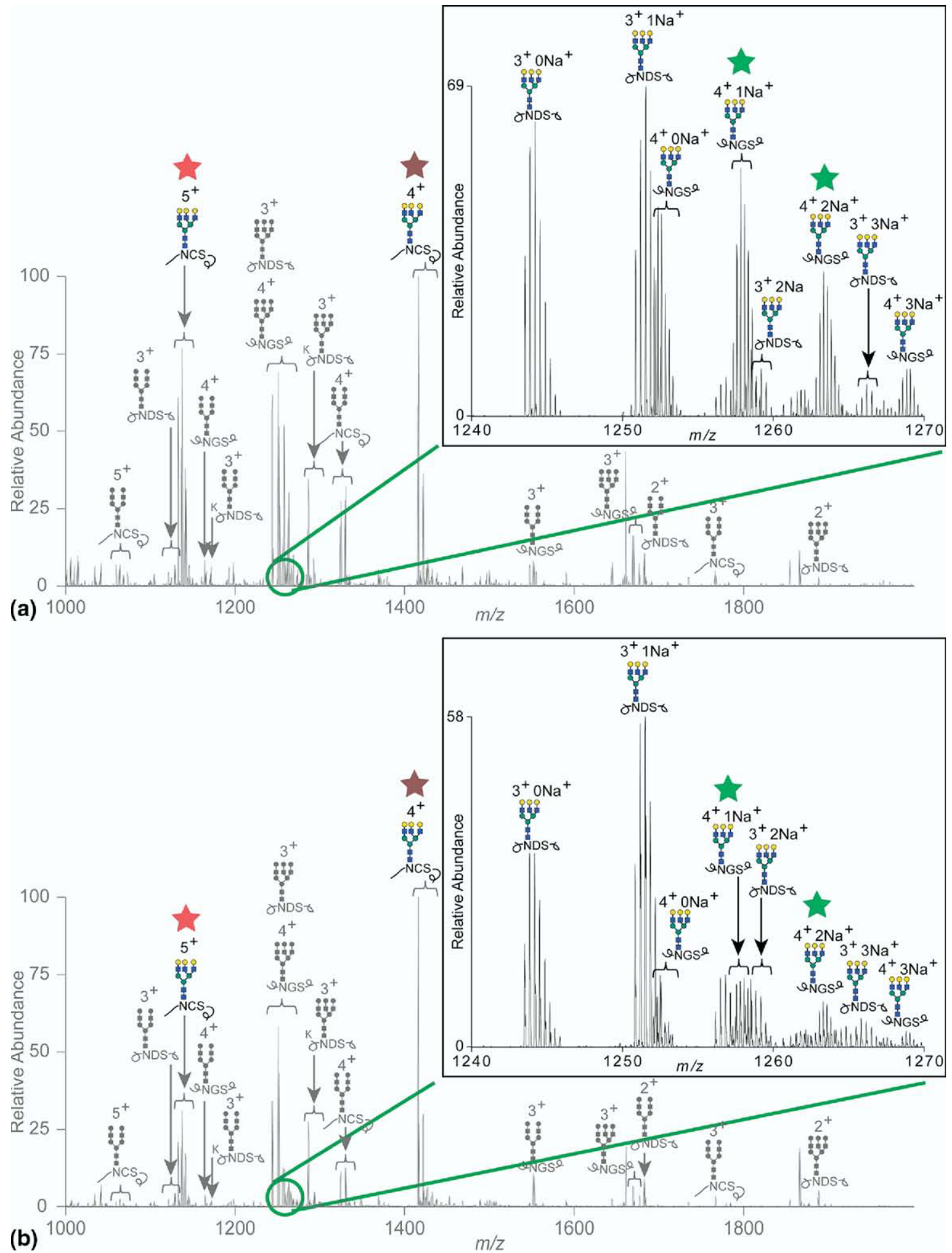

Figure 1. (a) and (b) Mass spectra from two different glycopeptide samples of asialofetuin. Red and brown stars show the same glycopeptide in different charge states; the intensities of each of the charge states vary from sample to sample. Insets: a zoomed-in region of the spectrum containing multiple sodiated adducts of the glycopeptides. The green stars indicate sodiated adducts of glycopeptides whose intensity changes in spectrum $\mathbf{a}$ and $\mathbf{b}$.

would have poor reproducibility when compared by their relative abundance alone.

To alleviate the problem that changes in the form in which the peaks ionize leads to irreproducible peak intensity data, the relative abundances of the peaks containing the same glycopeptide composition but differing in the charge state and charge carrier were combined. By combining these values, the problem that 
the peaks partition differently into different charge states is mitigated. Once all the charge states and charge carriers for a particular glycopeptide composition are combined, we report the results as a percent of the total glycopeptide ion signal, and this value is henceforth referred to as the glycopeptide percentage. [Reporting the values as percentages of all the glycopeptides present in the spectrum is a normalization method used to mitigate the run-to-run ionization discrepancies that are problematic in label-free quantitative approaches, as described in the introduction.] After obtaining the glycopeptide percentages for each of the different glyco- peptide compositions, the compositions are ordered based on their percentage from smallest to largest (rank ordered), so an internal comparison of the glycan profile can be made. A list of all the identified glycopeptide ions from asialofetuin are shown in Table 2, and the chart is color-coded to show which species' ion abundances were combined. For example, all the blue entries correspond to glycopeptides with the peptide sequence RPTGEVYDIEIDTLETTCHVLDPTPLANCSVR. The darker blue indicates the biantennary glycans containing that amino acid sequence, and the lighter blue indicates the triantennary glycans. The intensities of

Table 2. Glycopeptide peaks identified in asialofetuin FT-MS data

\begin{tabular}{|c|c|c|c|c|c|c|}
\hline Theoretical $\mathrm{m} / \mathrm{z}$ & Observed $\mathrm{m} / \mathrm{z}$ & $\begin{array}{l}\text { Mass } \\
\text { error }^{1}\end{array}$ & $\begin{array}{c}\text { Charge } \\
\text { state }\end{array}$ & $\begin{array}{c}\text { \# of } \\
\mathrm{Na}\end{array}$ & Peptide & Carbohydrate \\
\hline 1059.6757 & 1059.6767 & 0.9 & $5^{+}$ & 0 & RPTGEVYDIEIDTLETTCHVLDPTPLANCSVR & {$[\mathrm{Hex}] 5[\mathrm{HexNAc}] 4$} \\
\hline 1064.0722 & 1064.0783 & 5.7 & $5^{+}$ & 1 & “ & “ \\
\hline 1068.4687 & 1068.4746 & 5.5 & $5^{+}$ & 2 & “ & “ \\
\hline 1121.8122 & 1121.8183 & 5.4 & $3^{+}$ & 0 & LCPDCPLLAPLNDSR & “ \\
\hline 1129.1397 & 1129.1438 & 3.6 & $3^{+}$ & 1 & " & “ \\
\hline 1132.7022 & 1132.7059 & 3.3 & $5^{+}$ & 0 & RPTGEVYDIEIDTLETTCHVLDPTPLANCSVR & {$[\mathrm{Hex}] 6[\mathrm{HexNAc}] 5$} \\
\hline 1137.0987 & 1137.1055 & 6.0 & $5^{+}$ & 1 & “ & “ \\
\hline 1141.4952 & 1141.5009 & 5.0 & $5^{+}$ & 2 & “ & “ \\
\hline 1145.8917 & 1145.8944 & 2.4 & $5^{+}$ & 3 & “ & “ \\
\hline 1150.2882 & 1150.2762 & 10.4 & $5^{+}$ & 4 & “ & “ \\
\hline 1160.5443 & 1160.5529 & 7.4 & $4^{+}$ & 0 & VVHAVEVALATFNAESNGSYLQLVEISR & {$[\mathrm{Hex}] 5[\mathrm{HexNAc}] 4$} \\
\hline 1164.5106 & 1164.5156 & 4.3 & $3^{+}$ & 0 & KLCPDCPLLAPLNDSR & “ \\
\hline 1166.0400 & 1166.0479 & 6.8 & $4^{+}$ & 1 & VVHAVEVALATFNAESNGSYLQLVEISR & “ \\
\hline 1171.5356 & 1171.5389 & 2.8 & $4^{+}$ & 2 & 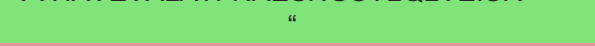 & “ \\
\hline 1171.8381 & 1171.8426 & 3.8 & $3^{+}$ & 1 & KLCPDCPLLAPLNDSR & “ \\
\hline 1243.5230 & 1243.5300 & 5.6 & $3^{+}$ & 0 & LCPDCPLLAPLNDSR & {$[\mathrm{Hex}] 6[\mathrm{HexNAc}] 5$} \\
\hline 1250.8505 & 1250.8559 & 4.3 & $3^{+}$ & 1 & “ & “ \\
\hline 1251.8274 & 1251.8252 & 1.8 & $4^{+}$ & 0 & VVHAVEVALATFNAESNGSYLQLVEISR & “ \\
\hline 1257.3230 & 1257.3286 & 4.5 & $4^{+}$ & 1 & “ & “ \\
\hline 1258.1780 & 1258.1814 & 2.7 & $3^{+}$ & 2 & LCPDCPLLAPLNDSR & “ \\
\hline 1262.8186 & 1262.8220 & 2.7 & $4^{+}$ & 2 & VVHAVEVALATFNAESNGSYLQLVEISR & “ \\
\hline 1265.5055 & 1265.5081 & 2.1 & $3^{+}$ & 3 & LCPDCPLLAPLNDSR & “ \\
\hline 1268.3143 & 1268.3221 & 6.1 & $4^{+}$ & 3 & VVHAVEVALATFNAESNGSYLQLVEISR & “ \\
\hline 1273.8099 & 1273.8070 & 2.3 & $4^{+}$ & 4 & " & “ \\
\hline 1286.2213 & 1286.2286 & 5.7 & $3^{+}$ & 0 & KLCPDCPLLAPLNDSR & “ \\
\hline 1293.5488 & 1293.5582 & 7.3 & $3^{+}$ & 1 & “ & “ \\
\hline 1300.8763 & 1300.8730 & 2.5 & $3^{+}$ & 2 & “ & “ \\
\hline 1308.2038 & 1308.2108 & 5.4 & $3^{+}$ & 3 & “ & “ \\
\hline 1324.3428 & 1324.3526 & 7.4 & $4^{+}$ & 0 & RPTGEVYDIEIDTLETTCHVLDPTPLANCSVR & {$[\mathrm{Hex}] 5[\mathrm{HexNAc}] 4$} \\
\hline 1329.8385 & 1329.8471 & 6.5 & $4^{+}$ & 1 & " & “ \\
\hline 1335.3341 & 1335.3426 & 6.4 & $4^{+}$ & 2 & “ & “ \\
\hline 1415.6259 & 1415.6355 & 6.8 & $4^{+}$ & 0 & “ & {$[\mathrm{Hex}] 6[\mathrm{HexNAc}] 5$} \\
\hline 1421.1215 & 1421.1297 & 5.8 & $4^{+}$ & 1 & “ & " \\
\hline 1426.6171 & 1426.6263 & 6.4 & $4^{+}$ & 2 & “ & “ \\
\hline 1432.1128 & 1432.1185 & 4.0 & $4^{+}$ & 3 & “ & “ \\
\hline 1437.6084 & 1437.6084 & 0.0 & $4^{+}$ & 4 & “ & “ \\
\hline 1547.0587 & 1547.0695 & 7.0 & $3^{+}$ & 0 & VVHAVEVALATFNAESNGSYLQLVEISR & {$[\mathrm{Hex}] 5[\mathrm{HexNAc}] 4$} \\
\hline 1554.3842 & 1554.3688 & 9.9 & $3^{+}$ & 1 & “ & “ \\
\hline 1668.7674 & 1668.7771 & 5.8 & $3^{+}$ & 0 & “ & {$[\mathrm{Hex}] 6[\mathrm{HexNAc}] 5$} \\
\hline 1676.0949 & 1676.1073 & 7.4 & $3^{+}$ & 1 & “ & “ \\
\hline 1682.2147 & 1682.2204 & 3.4 & $2^{+}$ & 0 & LCPDCPLLAPLNDSR & {$[\mathrm{Hex}] 5[\mathrm{HexNAc}] 4$} \\
\hline 1683.4224 & 1683.4358 & 8.0 & $3^{+}$ & 2 & VVHAVEVALATFNAESNGSYLQLVEISR & {$[\mathrm{Hex}] 6[\mathrm{HexNAc}] 5$} \\
\hline 1765.4547 & 1765.4575 & 1.6 & $3^{+}$ & 0 & RPTGEVYDIEIDTLETTCHVLDPTPLANCSVR & {$[\mathrm{Hex}] 5[\mathrm{HexNAc}] 4$} \\
\hline 1864.7808 & 1864.7876 & 3.6 & $2^{+}$ & 0 & LCPDCPLLAPLNDSR & {$[\mathrm{Hex}] 6[\mathrm{HexNAc}] 5$} \\
\hline
\end{tabular}

\footnotetext{
${ }^{1}$ Mass error is reported in ppm.
}

The color of the rows (blue, red, or green) represent the three glycosylation sites of asialofetuin, whereas the darker and lighter shades of a given color correspond to biantennary and triantennary glycans, respectively. Ion abundances corresponding to the same color and shade are added together to generate the "glycopeptide percentage" for each species. 
peaks with the same color are combined to produce a single glycopeptide percentage.

Figure 2 illustrates the benefit of combining the different charge states and charge carriers for the asialofetuin glycopeptide data. Figure 2a shows a portion of the rank order of ion abundances between the two asialofetuin samples from Figure 1 when the ion abundances are not combined, before ranking the glycopeptides from smallest to largest. The rank order is clearly very different between the two samples. However, once the different charge states and charge carriers for each of the glycopeptide compositions are combined, the rank order is highly reproducible between the two samples (Figure 2b).

\section{Quality Control Experiment 1-Robustness}

Since the goal was to develop a robust quantitation method that can detect changes in glycopeptides' intensities for different samples, one important feature is that the method must produce the same results for the same

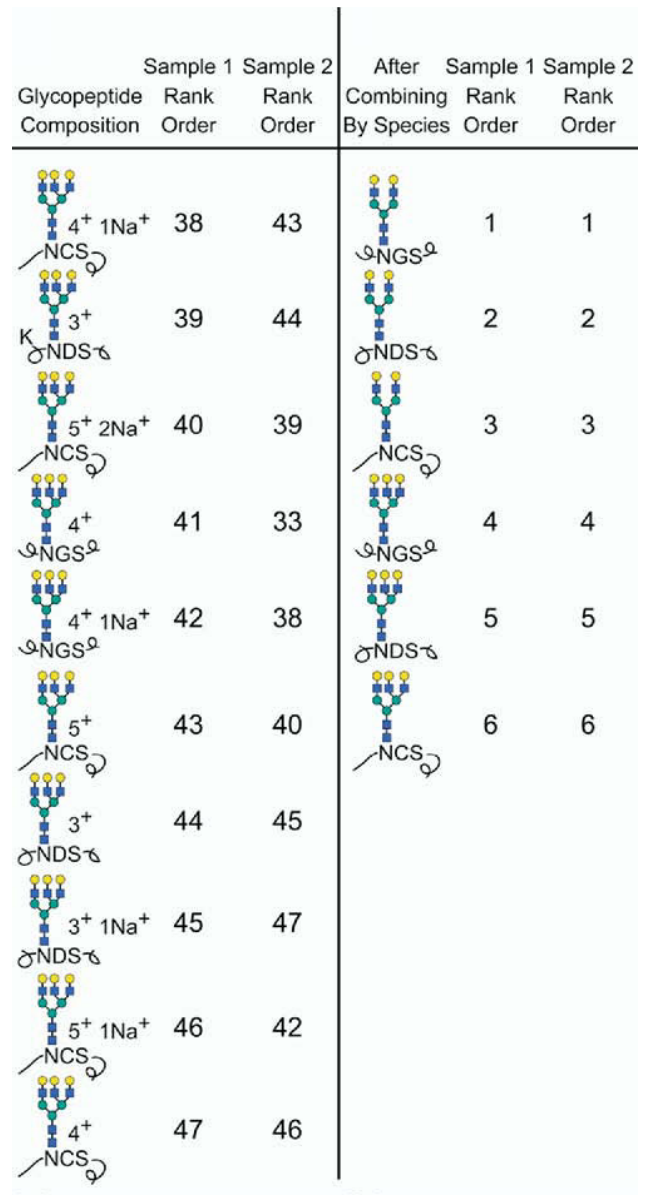

(a) glycoprotein batch. For example, small differences that could be introduced during digestion or glycopeptide enrichment and dilution for MS analysis, should not cause changes in the quantitation results. Otherwise, the method would not be robust. To test robustness, a quality control experiment was developed where four replicate samples of asialofetuin were each digested and prepared separately. After each sample was subjected to MS analysis, the glycopeptide percentages were calculated as described above, and were subsequently rank ordered, by percentage, from smallest to largest. The results of this experiment are displayed in Figure 3a. It is evident from the figure that the same rank order was observed for all four samples. However, the percentages between each sample tended to vary slightly, albeit not enough to change the rank order. This variability in percentage of asialofetuin glycopeptides is attributed to change in the distribution of sodiated adducts between samples. Different numbers of sodiated adducts can have slightly different ionization efficiencies. Therefore, when samples produce spectra with different intensities of sodiated adducts, combining the percentages of the different sodiated adducts can introduce a small variability in the percentages of each glycopeptide composition. This is not a significant problem, however, because the changes are small enough so that the rank order did not vary between the samples.

\section{Quality Control Experiment 2-Applicability to Different Glycoproteins}

Once the method was confirmed to produce reproducible results for asialofetuin, a second glycoprotein with very different properties than asialofetuin was analyzed to ensure the method is applicable for a wide variety of glycoproteins. RNase B was chosen for the analysis because it is much smaller than asialofetuin; it has only one glycosylation site; and it has a different type of glycosylation, high mannose type glycans, see Table 3. RNase B was subjected to the same sample preparation conditions as asialofetuin. Four replicate samples of RNase B were digested and analyzed. Unlike the asialofetuin data, RNase B glycopeptides did not ionize as sodiated adducts. The RNase B glycopeptides did ionize in multiple charge states, and the spectra also contained peaks corresponding to missed tryptic cleavages of the glycopeptides (data not shown). The missed cleavages are likely the result of the glycosylation blocking the cleavage site, as described earlier [44], since several arginine and lysine residues are located very near the glycosylation site. See the amino acid sequence in Table 3 . These missed tryptic cleavages could potentially interfere with reproducible quantitative analysis, if the digestions do not generate identical proportions of peptides with missed tryptic cleavages near the glycosylation sites. To mitigate the potential for quantitative error due to differences in the digestion,

Figure 2. (a) Rank order of glycopeptide ions from the data in Figure one. (b) Rank order of glycopeptide compositions, after combining all charge states and sodium adducts of ions with the same composition, before rank-ordering the components. Reproducible glycosylation profiles are achieved only in (b), when charge states and sodium adducts of the same species are combined. 

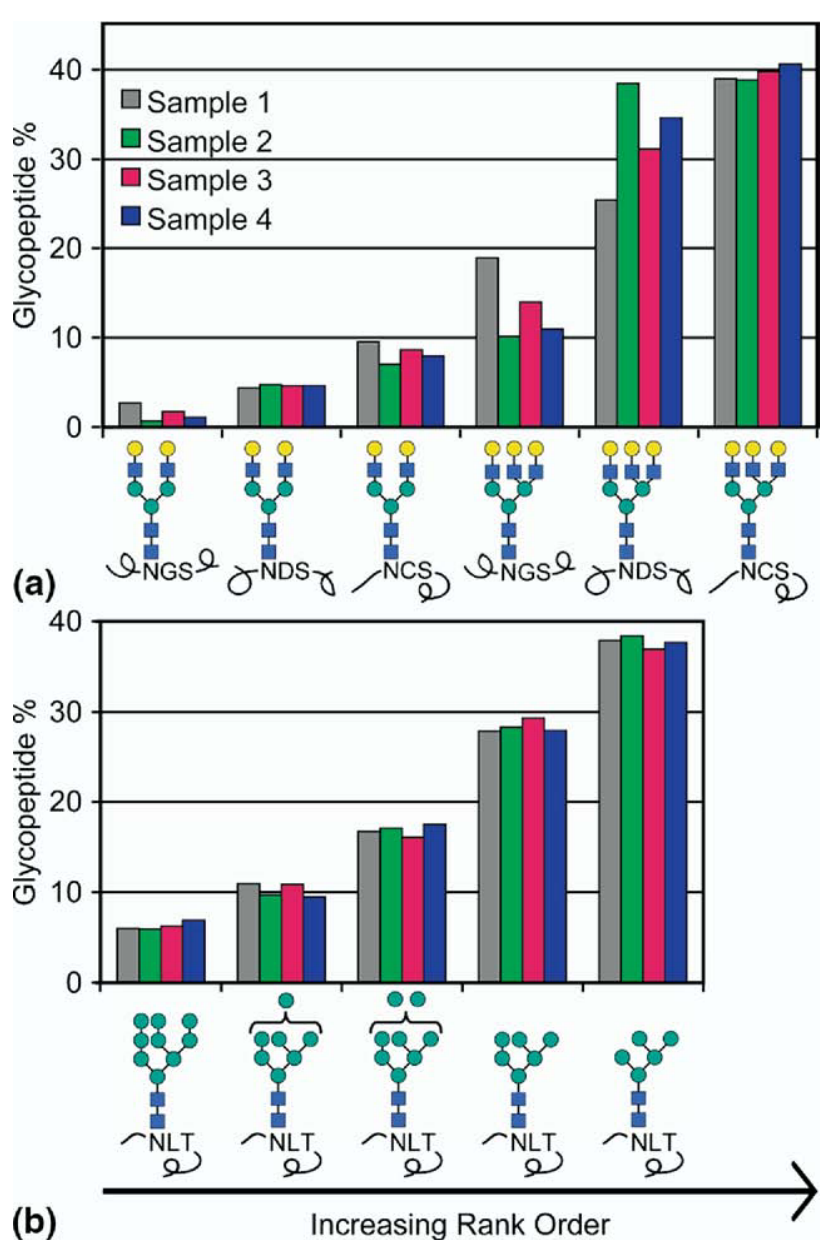

Figure 3. Similarity of the glycopeptide profiles for asialofetuin (a) and RNase B (b). The percentage of each glycopeptide composition is plotted for four replicate samples. The glycopeptide compositions are shown on the x-axis in order of their abundance in the spectrum. In each case, the rank order (smallest percentage to largest percentage) does not change; small fluctuations are observed in the actual percentage of each glycopeptide composition among the four replicate samples.

glycopeptide peaks corresponding to a given glycosylation site and a given glycan composition were combined with species that contained the same glycan composition and glycosylation site, but different levels of missed tryptic cleavages. The results from the RNase
B experiments are illustrated in Figure $3 b$. The RNase B glycopeptide data also exhibits a consistent rank order among the replicate samples.

\section{Quality Control Experiment 3-Instrument Precision}

The use of a mass spectrometer over extended time frames can lead to reproducibility problems in labelfree quantitative assays. Therefore, to ensure that minor changes in the instrument conditions do not lead to inaccuracies in assigning the rank order of glycopeptides, the four samples of glycopeptides from RNase B were run on two different dates, eight weeks apart. Between analyses, the samples were stored at $-20^{\circ} \mathrm{C}$. Under these conditions, the RNase B glycopeptides do not degrade; therefore any changes in the rank order of the glycopeptide compositions would be attributed to instrument variability over time [Dalpathado, D. S.; Irungu, J.; Zhang, Y.; Go, E. P.; Desaire, H., unpublished data]. Before using our quantitative method on the two sets of data, we first determined whether or not the mass spectra showed deviations in peak intensities between weeks zero and eight, by comparing the raw ion abundances for each of the glycopeptides. Table 4 shows the results for the relative abundances of several peaks that were acquired from one RNase B glycopeptide sample, before and after the 8-wk storage conditions. The relative abundance increases in the $3^{+}$charge state in week 8 compared to week 0 , while the relative abundance in the $2^{+}$charge state decreases in week 8 , compared to week 0 . This is another example that shows the ions can partition themselves differently into different charge states. In this case, drastically different ion abundances were acquired for the exact same sample. Fortunately, the quantitative method described herein is designed to accommodate this variability by combining glycopeptide peak percentages for the same glycopeptide, partitioned into different charge states. After combining the glycopeptide peak percentages as described above, the rank order of RNase B glycopeptides was obtained, and the data is shown in Figure 4 for the two different time points. The glycopeptide percentages for RNase B glycopeptides illustrated in

Table 3. Comparison of glycoproteins

\begin{tabular}{ll}
\hline \multicolumn{1}{c}{ RNase B } & \multicolumn{1}{c}{ Asialofetuin } \\
\hline \hline MALKSLVLLSLVLVLLLRVQPSLGKETAAAKFERQHMDSSTS & MKSFVLLFCLAQLWGCHSIPLDPVAGYKEPACDDPDTEQAALAAVD \\
AASSSNYCNQMMKSRNLTKDRCKPVNTFVHESLADVQAVC & YINKHLPRGYKHTLNQIDSVKVWPRRPTGEVYDIEIDTLETTCHVLD \\
SQKNVACKNGQTNCYQSYSTMSITDCRETGSSKYPNCAYKT & PTPLANCSVRQQTHAVEGDCDIHVLKQDGFSVLTKCDSS \\
TQANKHIIVACEGNPYVPVHFDASV & PDSAEDVRKLCPDCPLLAPLNDSRVVHAVEVALATFNAESNGSYL \\
& QLVEISRAQFVPLPVSVSVEFAVAATDCIAKEVVDPTKCNLLAEKQ \\
& YGFCKGSVIQKALGGEDVRVTCTLFQTQPVIPQPQPDGAEAEAPS \\
& AVPDAAGPTPSAAGPPVASVVGPSVVAVPLPLHRAHYDLRH \\
$16.5 \mathrm{kDa}$ & TFSGVASVESSSGEAFHVGKTPIVGQPSIPGGPVRLCPGRIRYFKI \\
$1 \mathrm{~N}$-linked glycosylation site & $38.5 \mathrm{kDa}$ \\
High mannose glycans & $3 \mathrm{~N}$-linked glycosylation sites \\
& Complex glycans \\
\hline
\end{tabular}


Table 4. Differences in relative abundance for glycopeptide ions from RNase B*

\begin{tabular}{|c|c|c|c|c|}
\hline Charge state & Composition & $m / z$ & Relative abundance week 0 & Relative abundance week 8 \\
\hline $3^{+}$ & {$[\mathrm{Hex}] 5[\mathrm{HexNAc}] 2+\mathrm{SRNLTKDR}$} & 735.9958 & 32.7 & 77.6 \\
\hline $3^{+}$ & {$[\mathrm{Hex}] 6[\mathrm{HexNAc}] 2+\mathrm{SRNLTKDR}$} & 790.0134 & 34.2 & 66.8 \\
\hline $3^{+}$ & {$[\mathrm{Hex}] 7[\mathrm{HexNAc}] 2+\mathrm{SRNLTKDR}$} & 844.0310 & 14.8 & 31.1 \\
\hline $3^{+}$ & {$[$Hex]8[HexNAc] $2+$ SRNLTKDR } & 898.0486 & 36.1 & 50.8 \\
\hline $3^{+}$ & {$[\mathrm{Hex}] 9[\mathrm{HexNAc}] 2+\mathrm{SRNLTKDR}$} & 952.0662 & 16.1 & 21.0 \\
\hline $2^{+}$ & {$[\mathrm{Hex}] 5[\mathrm{HexNAc}] 2+\mathrm{SRNLTKDR}$} & 1103.4899 & 67.4 & 40.0 \\
\hline $2^{+}$ & {$[\mathrm{Hex}] 6[\mathrm{HexNAc}] 2+\mathrm{SRNLTKDR}$} & 1184.5163 & 30.1 & 15.7 \\
\hline $2^{+}$ & {$[\mathrm{Hex}] 7[\mathrm{HexNAc}] 2+\mathrm{SRNLTKDR}$} & 1265.5427 & 6.4 & 3.3 \\
\hline $2^{+}$ & {$[\mathrm{Hex}] 8[\mathrm{HexNAc}] 2+\mathrm{SRNLTKDR}$} & 1346.5691 & 5.7 & 3.8 \\
\hline $2^{+}$ & {$[\mathrm{Hex}] 9[\mathrm{HexNAc}] 2+\mathrm{SRNLTKDR}$} & 1427.5955 & 0.8 & 0.6 \\
\hline
\end{tabular}

* Two data sets using the same sample, analyzed 8 weeks apart.

Figure 4 show high reproducibility, small standard deviations, and the rank order is retained between the two runs.

The above experiments demonstrate that for a purified sample, the quantitative method described produces reproducible data, even under different instrumental conditions, and the reproducibility is unaltered after repeating the protease digestion and sample preparation conditions. Also, the method successfully analyzed two different glycoproteins that had a variety of different features, including varying numbers of glycosylation sites and different types of glycosylation. In summary, these studies demonstrate that the quantitative method described would be useful for classifying glycosylation changes in purified proteins, which is useful in a variety of biopharmaceutical applications, as described in the introduction.

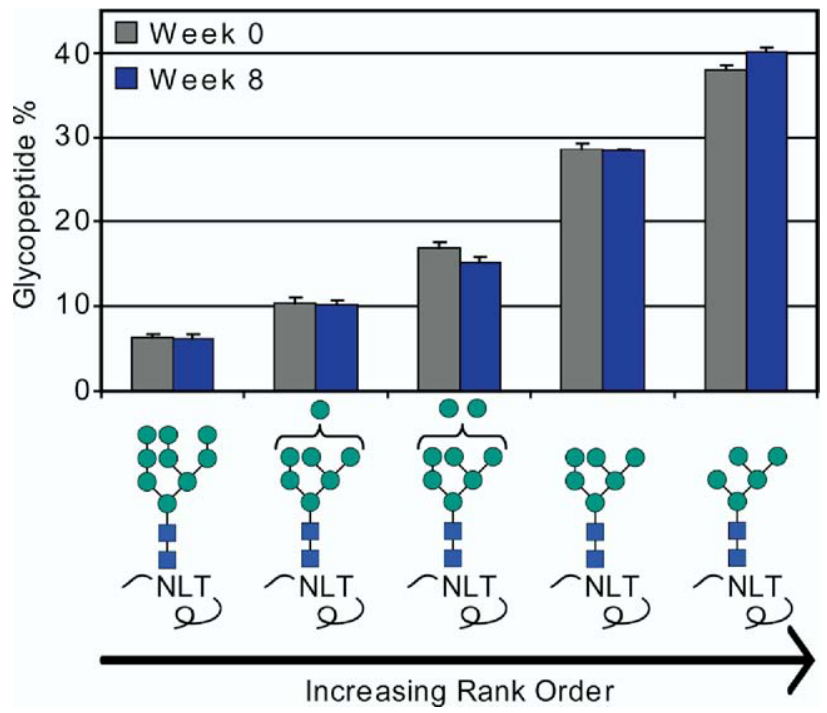

Figure 4. Graphical depiction of the quantified amounts of RNase B glycopeptides at two different time points. The mean from four digest samples was plotted. Error bars indicate the standard deviation. The glycopeptides are plotted from left to right in order of increasing abundance. The rank order does not change, even when the sample is re-analyzed 8 weeks after the original analysis date.

\section{Mixture Analysis}

In addition to characterizing the glycosylation on biopharmaceuticals, it would be ideal if this method of quantifying glycosylation profiles could also be used in other types of applications, for example in studies where a mixture of proteins is present. When a mixture of glycopeptides is analyzed, one current roadblock is being able to determine why the particular glycopeptide ion has changed in abundance, i.e., is it due to changes in glycosylation on a given protein or due to changes in the protein's concentration, relative to the other species being analyzed [4]? Our method, which characterizes the entire glycosylation profile for a given glycoprotein as part of the quantitation process, is ideally suited to solving this problem.

To demonstrate that the quantitative method described herein can also distinguish between glycosylation changes and net protein abundance changes when proteins are present in a mixture, glycopeptides from the two proteins described above were combined in several different ratios, and the resulting samples were analyzed. In each case, glycopeptides from asialofetuin were present at a concentration of $1 \mu \mathrm{M}$, while the glycopeptides from RNase B were present at varying concentrations, between 1 and $10 \mu \mathrm{M}$ (see Table 1). MS data of the mixed samples was acquired, and the rank order for each of the glycopeptides present was obtained, as described previously. The quantitative results are shown in Figure 5a. This figure shows data for the RNase B glycopeptides for the four samples. Regardless of the relative concentrations of the two proteins, the rank order of the glycans for the given glycoprotein, RNase B, did not change. In every case, the Man9 glycopeptides from RNase B were present in lowest abundance and the Man5 glycopeptides were present in highest abundance, among the RNase B glycoforms. This demonstrates that varying the concentration of protein does not impact the rank order of the glycopeptides for a given glycoprotein. In addition, the order observed in Figure 5a is the same order as observed for RNase B alone, (in Figure $3 \mathrm{~b}$ and 4), demonstrating that the presence of other proteins does not interfere with 

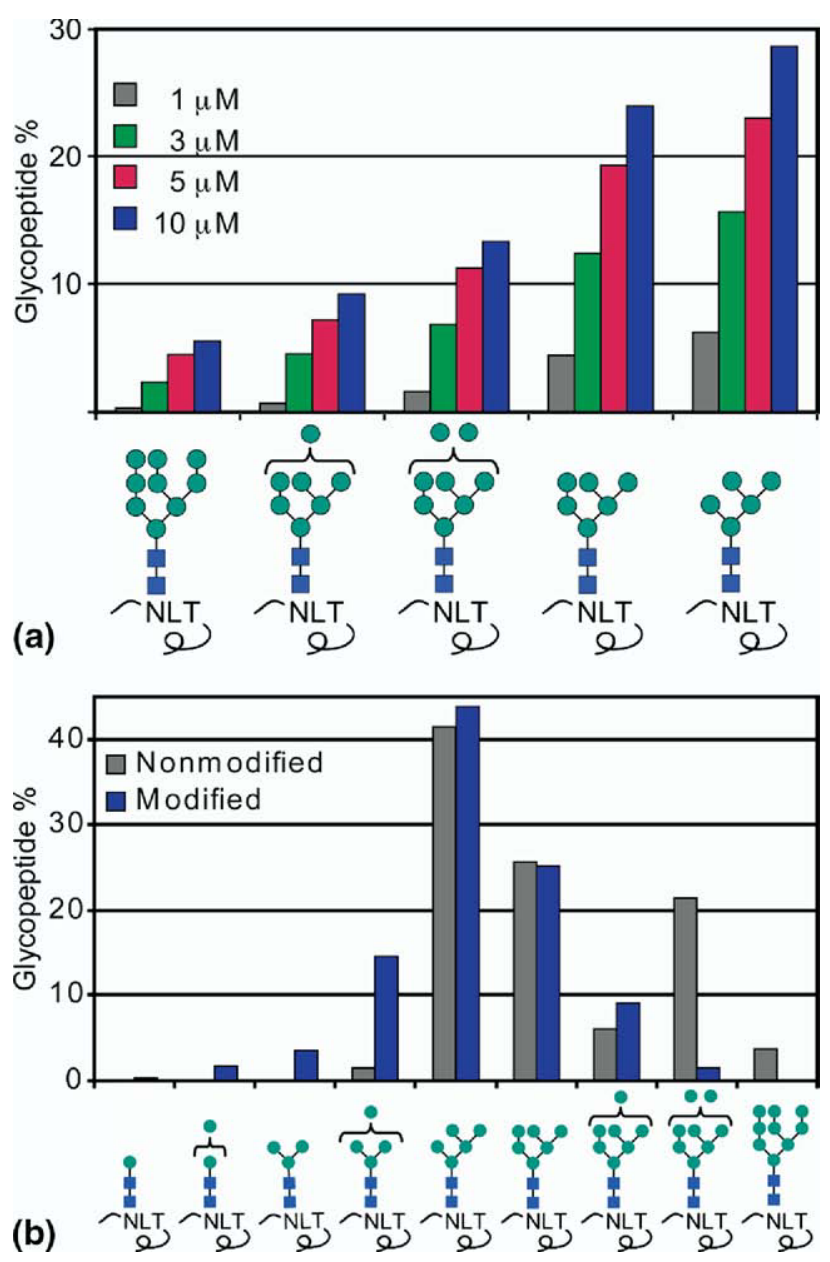

Figure 5. (a) Glycopeptide profiles for RNase B in mixtures of RNase B and asialofetuin. Glycopeptide percentages of all the RNase B glycoforms are increased as the concentration of RNase B is increased in the mixture; rank order of the glycoforms is conserved. (b) RNase B before and after cleavage with $\alpha$-mannosidase. Man8 and Man9 decreased in glycopeptide percentage, while Man1, Man2, Man3, and Man4 increased in glycopeptide percentage upon treatment with the enzyme. (Note: The glycans are plotted on the $X$ axis in (b) based on the number of mannose residues in the glycan, not based on increasing rank order. However, it is still visually evident from the graph that the two samples in (b), did not have a consistent rank order for their glycans.)

the method's ability to reproducibly rank order the glycopeptides. Most importantly, when one compares the data for a given glycopeptide, for example, Man5, the data in Figure 5a clearly demonstrate that the glycopeptide percentages increase as the concentration of the protein increases. Therefore, the data described herein can clearly distinguish between changes in a glycosylation profile and changes in a protein's concentration. For the four samples in Figure 5a, the glycosylation profile was identical; yet the concentrations of a given glycopeptide increased as the protein concentration increased.

It is important to note a rare case where this method would detect a false positive for protein concentration change: If a protein's glycosylation sites were only partially occupied in one sample, but fully occupied in another sample, the described method would incorrectly indicate that the protein with the fully occupied glycosylation sites had an increase in its concentration. In assessing protein concentration, this method does not account for the possibility of partially occupied glycosylation sites. If one needs to distinguish between protein concentration change and a change in glycosylation site occupancy, these two cases could easily be distinguished by determining the glycosylation site occupancy of the proteins in a separate experiment.

\section{Monitoring Changes in Glycosylation}

To demonstrate that the method can effectively detect changes in glycan population, the glycans on RNase B were modified by cleaving mannose residues with the enzyme, $\alpha$-mannosidase from Canavalia ensiformis. Two experiments were performed simultaneously; one where RNase B was allowed to be digested with $\alpha$-mannosidase and a second experiment with RNase B unmodified. Both samples were subjected to the same sample preparation and data analysis conditions. Under these conditions, the modified glycoprotein should have fewer mannoses present, since the enzyme cleaves mannose residues. Figure $5 \mathrm{~b}$ illustrates the results from this experiment. The figure shows that the protein with modified glycosylation had reduced glycopeptide percentages for Man8 and Man9, and the glycopeptide percentages for Man1, Man2, Man3, and Man4 increased, as expected. This clearly shows that the quantitative method is capable of detecting changes in glycosylation.

This quantitative method is similar to any other analytical method in that it has limitations. First of all, this method is only applicable to quantifying glycopeptides that have been previously characterized. It is expected that other methods would be used to identify all the glycopeptides present, before using the quantitative method. Appropriate complementary techniques for identifying glycopeptide compositions are welldescribed in the literature [12, 17, 20, 21, 40, 43, 45, 46]. The second major limitation of this method is that it is only applicable to glycoproteins containing neutral glycoforms; a mixture of sialylated glycoforms, or other negatively charged species, and neutral glycans typically requires the use of both positive and negative mode to accurately identify the glycoforms present [40]. This method does not currently allow for calculating glycosylation profiles from a combination of positive mode and negative mode data. Finally, as described above, the method cannot distinguish between changes in glycosylation site occupancy and glycoprotein concentration changes. Even though this method has some limitations, its utility for quantifying glycosylation profiles has many uses in glycoprotein analysis. 


\section{Conclusion}

This article describes a new label-free quantitative method that can be applied to purified proteins as well as glycoprotein mixtures. The method is able to distinguish between glycoprotein concentration changes and changes in glycosylation. The method was validated with several control experiments. The first control experiment analyzed replicate samples of glycopeptides from one glycoprotein, asialofetuin. The results from this experiment illustrate that the rank order of glycopeptides is consistent in all replicate samples, with slight variation in glycopeptide percentages. To be confident that the method would be applicable to a large set of glycoproteins, a second glycoprotein, RNase $B$, with very different properties, was analyzed. RNase $B$ results are also very consistent among four replicate samples, and the rank order of the glycoforms is retained among the replicate samples. Because the replicate samples in the two glycoproteins studied were digested in different vials before analysis, the results demonstrated that minor changes in digestion conditions did not alter the rank order. The third quality control experiment measured the ability of the method to tolerate small changes in the instrument conditions. The same RNase B glycopeptide samples were run on two different dates, 8 wk apart, and subsequently analyzed with the quantitative method. Similar RNase B glycopeptide percentages were observed, with no change in rank order; therefore similar samples can confidently be analyzed by this method at different times. This new quantitative method would be useful for anyone studying glycosylation profiles of proteins, either as purified proteins (as in the case of pharmaceutical development) or as glycoprotein mixtures, such as in the search for glycan-based biomarkers.

\section{Acknowledgments}

The authors acknowledge the National Institutes of Health for funding (project number RO1GM077266), the National Science Foundation (project number 0645120), and the Analytical Proteomics Laboratory at the University of Kansas for instrument time on the LIT-FTICR-MS.

\section{References}

1. Budnik, B. A.; Lee, R. S.; Steen, J. A. Global Methods for Protein Glycosylation Analysis by Mass Spectrometry. Biochim. Biophys. Acta 2006, 1764, 1870-1880.

2. Morelle, W.; Canis, K.; Chirat, F.; Faid, V.; Michalski, J.-C. The Use of Mass Spectrometry for the Proteomic Analysis of Glycosylation. Proteomics 2006, 6, 3993-4015.

3. Hayes, B. K.; Freeze, H. H.; Varki, A. Biosynthesis of Oligosaccharides in Intact Golgi Preparations from Rat Liver. Analysis of N-linked Glycans by UDP-[6- $\left.{ }^{3} \mathrm{H}\right] \mathrm{N}$-acetylglucosamine. J. Biol. Chem. 1993, 268, 16139-16154.

4. Zhao, J.; Qiu, W.; Simeone, D. M.; Lubman, D. M. N-linked Glycosylation Profiling of Pancreatic Cancer Serum Using Capillary Liquid Phase Separation Coupled with Mass Spectrometric Analysis. J. Proteome Res. 2007, 6, 1126-1138.

5. Orntoft, T. F.; Vestergaard, E. M. Clinical Aspects of Altered Glycosylation of Glycoproteins in Cancer. Electrophoresis 1999, 20, 362-371.

6. Kim, Y. J.; Varki, A. Perspectives on the Significance of Altered Glycosylation of Glycoproteins in Cancer. Glycoconj. J. 1997, 14, 569576.
7. Maiese, K.; Li, F.; Chong, Z. Z. Erythropoietin in the Brain: Can the Promise to Protect be Fulfilled? Trends Pharmacol. Sci. 2004, 25, 577-583.

8. Loumaye, E.; Dreano, M.; Galazka, A.; Howles, C.; Ham, L.; Munafo, A.; Eshkol, A.; Giudice, E.; De Luca, E.; Sirna, A.; Antonetti, F.; Giartosio, C.-E.; Scaglia, L.; Kelton, C.; Campbell, R.; Chappel, S. Recombinant Follicle Stimulating Hormone: Development of the First Biotechnology Product for the Treatment of Infertility. Hum. Reprod. Update 1998, 4, 862-881.

9. Szkudlinski, M. W.; Thotakura, N. R.; Bucci, I.; Joshi, L. R.; Tsai, A.; East-Palmer, J.; Shiloach, J.; Weintraub, B. D. Purification and Characterization of Recombinant Human Thyrotropin (TSH) Isoforms Produced by Chinese Hamster Ovary Cells: The Role of Sialylation and Sulfation in TSH Bioactivity. Endocrinology 1993, 133, 1490-1503.

10. Hamilton, S. R.; Davidson, R. C.; Sethuraman, N.; Nett, J. H.; Jiang Y.; Rios, S.; Bobrowicz, P.; Stadheim, T. A.; Li, H.; Choi, B.-K.; Hopkins, D.; Wischnewski, H.; Roser, J.; Mitchell, T.; Strawbridge, R. R.; Hoopes, J.; Wildt, S.; Gerngross, T. U. Humanization of Yeast to Produce Complex Terminally Sialylated Glycoproteins. Science 2006, 313, 1441-1443.

11. Burton, D. R.; Desrosiers, R. C.; Doms, R. W.; Koff, W. C.; Kwong, P. D.; Moore, J. P.; Nabel, G. J.; Sodroski, J.; Wilson, I. A.; Wyatt, R. T. HIV Vaccine Design and the Neutralizing Antibody Problem. Nat. Immunol. 2004, 5, 233-236.

12. Go, E. P.; Irungu, J.; Zhang, Y.; Dalpathado, D. S.; Liao, H.-X.; Sutherland, L. L.; Alam, S. M.; Haynes, B. F.; Desaire, H. Glycosylation Site-Specific Analysis of HIV Envelope Proteins (JR-FL and CON-S) Reveals Major Differences in Glycosylation Site Occupancy, Glycoform Profiles, and Antigenic Epitopes' Accessibility. J. Proteome Res. 2008, 7, 1660-1674.

13. Gerngross, T. U. Advances in the Production of Human Therapeutic Proteins in Yeasts and Filamentous Fungi. Nat. Biotechnol. 2004, 22, 1409-1414.

14. Wong, C.-H. Protein Glycosylation: New Challenges and Opportunities. J. Org. Chem. 2005, 70, 4219-4225.

15. Wuhrer, M.; Catalina, M. I.; Deelder, A. M.; Hokke, C. H. Glycoproteomics Based on Tandem Mass Spectrometry of Glycopeptides. J. Chromatogr. B 2007, 849, 115-128.

16. Mechref, Y.; Novotny, M. V. Structural Investigations of Glycoconjugates at High Sensitivity. Chem. Rev. 2002, 102, 321-369.

17. Dalpathado, D. S.; Desaire, H. Glycopeptide Analysis by Mass Spectrometry. Analyst (Cambridge, U. K.) 2008, 133, 731-738.

18. Joenväärä, S.; Ritamo, I; Peltoniemi, H.; Renkonen, R. N-Glycoproteomics-An Automated Workflow Approach. Glycobiology 2008, 18, 339-349.

19. Varki, A. Biological Roles of Oligosaccharides: All of the Theories are Correct. Glycobiology 1993, 3, 97-130.

20. Dalpathado, D. S.; Irungu, J.; Go, E. P.; Nortan, K.; Bousfield, G. R.; Desaire, H. Comparative Glycomics of the Glycoprotein Follicle-Stimulating Hormone (FSH): Glycopeptide Analysis of Isolates from Two Mammalian Species. Biochemistry 2006, 45, 8665-8673.

21. Irungu, J.; Dalpathado, D. S.; Go, E. P.; Jiang, H.; Ha, H. V.; Bousfield G. R.; Desaire, H. A Method for Characterizing Sulfated Glycoproteins in a Glycosylation Site-Specific Fashion, Using Ion-Pairing and Tandem Mass Spectrometry. Anal Chem. 2006, 78, 1181-1190.

22. Patwa, T. H.; Zhao, J.; Anderson, M. A.; Simeone, D. M.; Lubman, D. M. Screening of Glycosylation Patterns in Serum Using Natural Glycoprotein Microarrays and Multi-Lectin Fluorescence Detection. Anal. Chem. 2006, 78, 6411-6421.

23. Pilobello, K. T.; Krishnamoorthy, L.; Slawek, D.; Mahal, L. K. Development of a Lectin Microarray for the Rapid Analysis of Protein Glycopatterns. Chem. Biochem. 2005, 6, 985-989.

24. Xiong, L.; Andrews, D.; Regnier, F. Comparative Proteomics of Glycoproteins Based on Lectin Selection and Isotope Coding. J. Proteome Res. 2003, 2, 618-625.

25. Zhang, H.; Li, X.-J.; Martin, D. B.; Aebersold, R. Identification and Quantification of N-linked Glycoproteins Using Hydrazide Chemistry, Stable Isotope Labeling and Mass Spectrometry. Nat. Biotechnol. 2003, $21,660-666$

26. Atwood, J. A., III; Cheng, L.; Alvarex-Manilla, G.; Warren, N. L.; York, W. S.; Orlando, R. Quantitation by Isobaric Labeling: Applications to Glycomics. J. Proteome Res. 2008, 7, 367-374.

27. Zhao, J.; Patwa, T. H.; Qiu, W.; Shedden, K.; Hinderer, R.; Misek, D. E. Anderson, M. A., Simeone, D. M.; Lubman, D. M. Glycoprotein Microarrays with Multi-Lectin Detection: Unique Lectin Binding Patterns as a Tool for Classifying Normal, Chronic Pancreatitis, and Pancreatic Cancer Sera. I. Proteome Res. 2007, 6, 1864-1874.

28. Alvarez-Manilla, G.; Warren, N. L.; Abney, T.; Atwood, J. III; Azadi, P. York, W. S.; Pierce, M.; Orlando, R. Tools for Glycomics: Relative Quantitation of Glycans by Isotopic Permethylation Using ${ }^{13} \mathrm{CH}_{3} \mathrm{I}$. Glycobiology 2007, 17, 677-687.

29. Uematsu, R.; Furukawa, J.-I.; Nakagawa, H.; Shinohara, Y.; Deguchi, K. Monde, K.; Nishimura, S.-I. High Throughput Quantitative Glycomics and Glycoform-Focused Proteomics of Murine Dermis and Epidermis. Mol. Cell. Proteom. 2005, 4, 1977-1989.

30. Kita, Y.; Miura, Y.; Furukawa, J.-I.; Nakano, M.; Shinohara, Y.; Ohno, M.; Takimoto, A.; Nishimura, S.-I. Quantitative Glycomics of Human Whole Serum Glycoproteins Based on the Standardized Protocol for Liberating N-Glycans. Mol. Cell. Proteom. 2007, 6, 1437-1445.

31. Furukawa, J.-I.; Shinohara, Y.; Kuramoto, H.; Miura, Y.; Shimaoka, H. Kurogochi, M.; Nakano, M.; Nishimura, S.-I. Comprehensive Approach to Structural and Functional Glycomics Based on Chemoselective Gly- 
coblotting and Sequential Tag Conversion. Anal. Chem. 2008, 80 , 1094-1101.

32. Wuhrer, M.; Stam, J. C.; van de Geijn, F. E.; Koeleman, C. A. M.; Verrips, C. T.; Dolhain, R. J. E. M.; Hokke, C. H.; Deelder, A. M. Glycosylation Profiling of Immunoglobulin G (IgG) Subclasses from Human Serum. Proteomics 2007, 7, 4070-4081.

33. Old, W. M.; Meyer-Arendt, K.; Aveline-Wolf, L.; Pierce, K. G.; Mendoza, A.; Sevinskey, J. R.; Resing, K. A.; Ahn, N. G. Comparison of Label-Free Methods for Quantifying Human Proteins by Shotgun Proteomics. Mol. Cell. Proteom. 2005, 4, 1487-1502.

34. Callister, S. J.; Barry, R. C.; Adkins, J. N.; Johnson, E. T.; Qian, W.-J.; Webb-Robertson, B.-J.; Smith, R. D.; Lipton, M. S. Normalization Approaches for Removing Systematic Biases Associated with Mass Spectrometry and Label-Free Proteomics. J. Proteome Res. 2006, 5, 277-286.

35. Ono, M.; Shitashige, M.; Honda, K.; Isobe, T.; Kuwabara, H.; Matsuzuki, H.; Hirohashi, S.; Yamada, T. Label-Free Quantitative Proteomics Using Large Peptide Data Sets Generated by Nanoflow Liquid Chromatography and Mass Spectrometry. Mol. Cell. Proteom. 2006, 5, 1338-1347.

36. Wang, W.; Zhou, H.; Lin, H.; Roy, S.; Shaler, T. A.; Hill, L. R.; Norton, S.; Kumar, P.; Anderle, M.; Becker, C. H. Quantification of Proteins and Metabolites by Mass Spectrometry without Isotopic Labeling or Spiked Standards. Anal. Chem. 2003, 75, 4818-4826.

37. Toumi, M. L.; Go, E. P.; Desaire, H. Development of Fully Functional Proteins with Novel Glycosylation Via Enzymatic Glycan Trimming. J. Pharm. Sci., in press.

38. Wada, Y.; Tajiri, M.; Yoshida, S. Hydrophilic Affinity Isolation and MALDI Multiple-Stage Tandem Mass Spectrometry of Glycopeptides for Glycoproteomics. Anal. Chem. 2004, 76, 6560-6565.
39. Tajiri, M.; Yoshida, S.; Wada, Y. Differential Analysis of Site-Specific Glycans on Plasma and Cellular Fibronectins: Application of a Hydrophilic Affinity Method for Glycopeptide Enrichment. Glycobiology 2005, 15, 1332-1340.

40. Zhang, Y.; Go, E. P.; Desaire, H. Maximizing Coverage of Glycosylation Heterogeneity in MALDI-MS Analysis of Glycoproteins with Up to 27 Glycosylation Sites. Anal. Chem. 2008, 80, 3144-3158.

41. Palm, A. K.; Novotny, M. V. A Monolithic PNGase F Enzyme Microreactor Enabling Glycan Mass Mapping of Glycoproteins by Mass Spectrometry. Rapid Commun. Mass Spectrom. 2005, 19, 1730-1738.

42. Rudd, P. M.; Joao, H. C.; Coghill, E.; Fiten, P.; Saunders, M. R.; Opdenakker, G.; Dwek, R. A. Glycoforms Modify the Dynamic Stability and Functional Activity of an Enzyme. Biochemistry 1994, 33, 17-22.

43. Irungu, J.; Go, E. P.; Zhang, Y.; Dalpathado, D. S.; Liao, H.-X.; Haynes, B. F.; Desaire, H. Comparison of HPLC/ESI-FTICR MS Versus MALDITOF/TOF MS for Glycopeptide Analysis of a Highly Glycosylated HIV Envelope Glycoprotein. J. Am. Soc. Mass Spectrom. 2008, 19, 1209-1220.

44. Imre, T.; Schlosser, G.; Pocsfalvi, G. Siciliano, R: Molnar-Szollosi, E. Kremmer, T.; Malorni, A.; Vekey, K. Glycosylation Site Analysis of Human Apha-1-acid Glycoprotein (AGP) by Capillary Liquid ChromatographyElectrospray Mass Spectrometry. J. Mass Spectrom. 2005, 40, 1472-1483.

45. Go, E. P.; Rebecchi, K. R.; Dalpathado, D. S.; Bandu, M. L.; Zhang, Y Desaire, H. GlycoPep DB: A Tool for Glycopeptide Analysis Using a "Smart Search." Anal. Chem. 2007, 79, 1708-1713.

46. Irungu, J.; Go, E. P.; Dalpathado, D. S.; Desaire, H. Simplification of Mass Spectral Analysis of Acidic Glycopeptides Using GlycoPep ID. Anal. Chem. 2007, 79, 3065-3074. 